\title{
Type 3 Neovascularization Associated with Retinitis Pigmentosa
}

\author{
Jihene Sayadi $^{a} \quad$ Alexandra Miere $^{a} \quad$ Eric H. Souied ${ }^{a}$ Salomon Y. Cohen ${ }^{a, b}$ \\ ${ }^{a}$ Department of Ophthalmology, Intercity Hospital and University Paris Est, Créteil, France; \\ ${ }^{\mathrm{b}}$ Ophthalmic Center for Imaging and Laser, Paris, France
}

\section{Keywords}

Type 3 neovascularization - Retinal choroidal anastomosis - Choroidal neovascularization · Retinal angiomatous proliferation · Retinitis pigmentosa · Cystoid macular edema - Optical coherence tomography angiography

\begin{abstract}
Purpose: To report a case of type 3 neovascular lesion in a patient with retinitis pigmentosa (RP) complicated by macular edema. Case Report: A 78-year-old man with a long follow-up for RP was referred for painless visual acuity decrease in the right eye. Best-corrected visual acuity was 20/125 in the right eye and 20/40 in the left. Fundus examination showed typical $\mathrm{RP}$ and macular edema in both eyes. In the right eye, spectral domain optical coherence tomography revealed a marked cystic macular edema associated with disruption of the Bruch membrane/retinal pigment epithelium complex overlying a pigmentary epithelium detachment, with a vascular structure which appeared to originate from the deep capillary plexus and to be connected with the subretinal pigment epithelium space. Optical coherence tomography angiography showed a high-flow vessel infiltrating the outer retinal layers in the deep capillary plexus segmentation, and a tuft-shaped, bright, high-flow network that seemed to be connected with the subretinal pigment epithelium space in the outer retinal layer segmentation. This presentation was consistent with an early type 3 neovascular lesion in the right eye. Conclusion: Type 3 neovascularization may be considered a possible complication of RP.




\section{Introduction}

Retinitis pigmentosa (RP) has rarely been associated with choroidal neovascularization (CNV) [1-6]. Furthermore, its association with type 3 neovascularization is even exceptional, and only 1 case of RP associated with type 3 neovascularization has been reported to date [6]. This study reports a type 3 neovascular lesion in the context of RP complicated by cystoid macular edema, diagnosed by noninvasive optical coherence tomography angiography (OCTA).

\section{Case Report}

A 78-year-old man was referred for painless visual acuity decrease in the right eye. The patient had a long-follow-up for RP. Best-corrected visual acuity was 20/125 in the right eye and 20/40 in the left. Anterior segment examination was unremarkable except for a mild cataract. Fundus photography and fundus autofluorescence were performed with a Topcon 50-TRX fundus camera (Topcon, Tokyo, Japan) using Spaide filters for autofluorescence. Fundus examination of both eyes revealed optic disc pallor, peripapillary atrophy, arteriolar attenuation, and typical bone spicules involving the mid-peripheral retina. In addition, a macular edema was present in both eyes, more prominent in the right eye. There was no hemorrhage and no sign of CNV.

Spectral domain optical coherence tomography (CIRRUS; Zeiss, Jena, Germany) revealed cystoid macular edema and epiretinal membrane in both eyes. However, in the nasal part of the fovea of the right eye, a disruption of the Bruch membrane/retinal pigment epithelium complex overlying a retinal pigment epithelium detachment was observed. It was associated with a vascular structure which appeared to originate from the deep capillary plexus and to be connected with the subretinal pigment epithelium space.

These findings supported a type 3 neovascularization in the right eye. Nevertheless, given the unusual clinical picture, we decided to perform OCTA (Optovue, Inc., Freemont, CA, USA). Fluorescein angiography was not performed in order to avoid exposure to strong light flash. OCTA showed a high-flow vessel infiltrating the outer retinal layers in the deep capillary plexus segmentation and a tuft-shaped, bright, high-flow network characterized by a retinal-retinal anastomosis in the outer retinal layer segmentation (Fig. 1).

Thanks to OCTA, a noninvasive diagnosis of type 3 neovascular lesion complicating an RP with cystoid macular edema could be made. The patient was subsequently scheduled for 3 consecutive monthly intravitreal injections of ranibizumab. He received 8 intravitreal injections during the first year of follow-up.

\section{Discussion}

RP comprises a group of inherited retinal diseases causing photoreceptor and retinal pigment epithelium degeneration. The disease is characterized by night blindness, midperipheral hyperpigmentary spots with a bone spicule aspect and arteriolar attenuation, visual field constriction, hypovolted electroretinogram waves, and slow progressive alteration of these signs [7]. The central vision is usually spared up to the later stages of the disease in patients with RP. However, during the course of the disease, macular complications may occur, leading to much earlier and often severe central visual impairment. The most 


\section{Case Reports in Ophthalmology}

frequent macular abnormalities in RP are cystoid macular edema, followed by epiretinal membrane [8]. Choroidal neovascular membrane is very rarely associated with RP. To our knowledge, only 8 cases of CNV have been described in the literature [1-6]. Seven of them were type $2 \mathrm{CNV}, 5$ had a subfoveal location, and 2 a juxtafoveal location. One case of RP with retinal angiomatous proliferation confirmed by indocyanine green angiogram has been reported in a 60-year-old woman [6]. Based on CNV therapies available at the time of their occurrence, CNVs complicating RP have been treated with laser photocoagulation [1], photodynamic therapy [3], and medical therapy [4, 5]. Intravitreal bevacizumab has been reported to be effective for choroidal neovascular membrane stabilization in patients with RP. A case of subfoveal CNV associated with RP was successfully managed with a single intravitreal bevacizumab injection [4]. However, in another report, 5 intravitreal injections were required over a 1-year period to achieve CNV stabilization, suggesting that anatomical stabilization of RP-related CNV may be difficult to achieve [5].

The present case corresponded to a type 3 neovascular lesion complicating an RP with cystoid macular edema. Type 3 neovascularization, also referred to as retinal angiomatous proliferation, is a type of new vessels which was described in 2008. It is a common form of neovascular age-related macular degeneration with a predilection for the neurosensory retina. It is characterized by a compensatory telangiectatic response associated with the intraretinal proliferative activity, leading to a retinal choroidal anastomosis [9]. Type 3 neovascularization has rarely been reported in conditions other than age-related macular degeneration such as childhood Coats disease, radiation retinopathy, and syphilitic retinitis [10-13]. Photoreceptor cell degeneration, concomitant retinal pigment epithelium changes, and choriocapillaris damage often found in RP could result in a proliferative activity within the neurosensory retina and lead to the formation of a retinal choroidal anastomosis.

OCTA is a tridimensional, rapid, noninvasive technique which has been described as a potentially interesting tool to assess type 3 neovascularization, with clear visualization of high-flow lesions in affected eyes $[14,15]$.

In conclusion, type 3 neovascularization may be considered a possible complication of RP. OCTA may avoid the use of invasive tools and allowed, in the present case, an easy diagnosis of type 3 neovascularization complicating the condition.

\section{Acknowledgment}

This work was supported in part by CIL-ASSOC, Association for Education and Research, Centre Ophtalmologique d'Imagerie et de Laser, Paris, France.

\section{Statement of Ethics}

The patient's informed consent has been obtained.

\section{Disclosure Statement}

The authors declare that they have no conflicts of interest to disclose. 


\section{Case Reports in Ophthalmology}

\begin{tabular}{l|l}
\hline DOI: $10.1159 / 000471790$ & C 2017 The Author(s). Published by S. Karger AG, Basel
\end{tabular}
www.karger.com/cop

Sayadi et al.: Type 3 Neovascularization Associated with Retinitis Pigmentosa

\section{References}

1 Marano F, Deutman AF, Leys A, Aandekerk AL: Hereditary retinal dystrophies and choroidal neovascularization. Graefes Arch Clin Exp Ophthalmol 2000;238:760-764.

-2 Iwakiri R, Okinami S, Hirata A: Two cases of retinitis pigmentosa associated with choroidal neovascularization. Nippon Ganka Gakkai Zasshi 2007;111:606-611.

-3 Cheng JY, Adrian KH: Photodynamic therapy for choroidal neovascularization in Stargardt disease and retinitis pigmentosa. Retin Cases Brief Rep 2009;3:388-390.

4 Malik A, Sood S, Narang S: Successful treatment of choroidal neovascular membrane in retinitis pigmentosa with intravitreal bevacizumab. Int Ophthalmol 2010;30:425-428.

-5 Battaglia Parodi M, De Benedetto U, Knutsson KA, Librando A, Bandello F, Lanzetta P, Iacono P: Juxtafoveal choroidal neovascularization associated with retinitis pigmentosa treated with intravitreal bevacizumab. J Ocul Pharmacol Ther 2012;28:202-204.

-6 Nagao Y, Hiramoto Y, Takahashi K: A case of retinitis pigmentosa with retinal angiomatous proliferation. Nippon Ganka Gakkai Zasshi 2011;115:147-150.

7 Baumgartner WA: Etiology, pathogenesis, and experimental treatment of retinitis pigmentosa. Med Hypotheses 2000;54:814-824.

-8 Testa F, Rossi S, Colucci R, Gallo B, Di Iorio V, della Corte M, Azzolini C, Melillo P, Simonelli F: Macular abnormalities in Italian patients with retinitis pigmentosa. Br J Ophthalmol 2014;98:946-950.

-9 Freund KB, Ho IV, Barbazetto IA, Koizumi H, Laud K, Ferrara D, Matsumoto Y, Sorenson JA, Yannuzzi L: Type 3 neovascularization: the expanded spectrum of retinal angiomatous proliferation. Retina 2008;28:201-211.

10 Quijano C, Querques G, Massamba N, Soubrane G, Souied EH: Type 3 choroidal neovascularization associated with fundus flavimaculatus. Ophthalmic Res 2009;42:152-154.

-11 Sigler EJ, Calzada JI: Retinal angiomatous proliferation with chorioretinal anastomosis in childhood Coats disease: a reappraisal of macular fibrosis using multimodal imaging. Retina 2015;35:537-546.

12 Mendrinos E, Pilly B, Baglivo E, Donati G, Safran AB, Pournaras CJ: Chorioretinal anastomosis as a rare complication of radiation retinopathy. Acta Ophthalmol 2009;87:473-475.

13 Rouvas A, Petrou P, Maselos S, Douvali M, Vergados I, Markomichelakis N: Retinal angiomatous proliferation (RAP) in a 42-year-old woman with syphilitic retinitis. Ocul Immunol Inflamm 2009;17: 361-363.

14 Miere A, Querques G, Semoun O, El Ameen A, Capuano V, Souied EH: Optical coherence tomography angiography in early type 3 neovascularization. Retina 2015;35:2236-2241.

15 Kuehlewein L, Dansingani KK, de Carlo TE, Bonini Filho MA, Iafe NA, Lenis TL, Freund KB, Waheed NK, Duker JS, Sadda SR, Sarraf D: Optical coherence tomography angiography of type 3 neovascularization secondary to age-related macular degeneration. Retina 2015;35:2229-2235. 


\section{Case Reports in Ophthalmology}
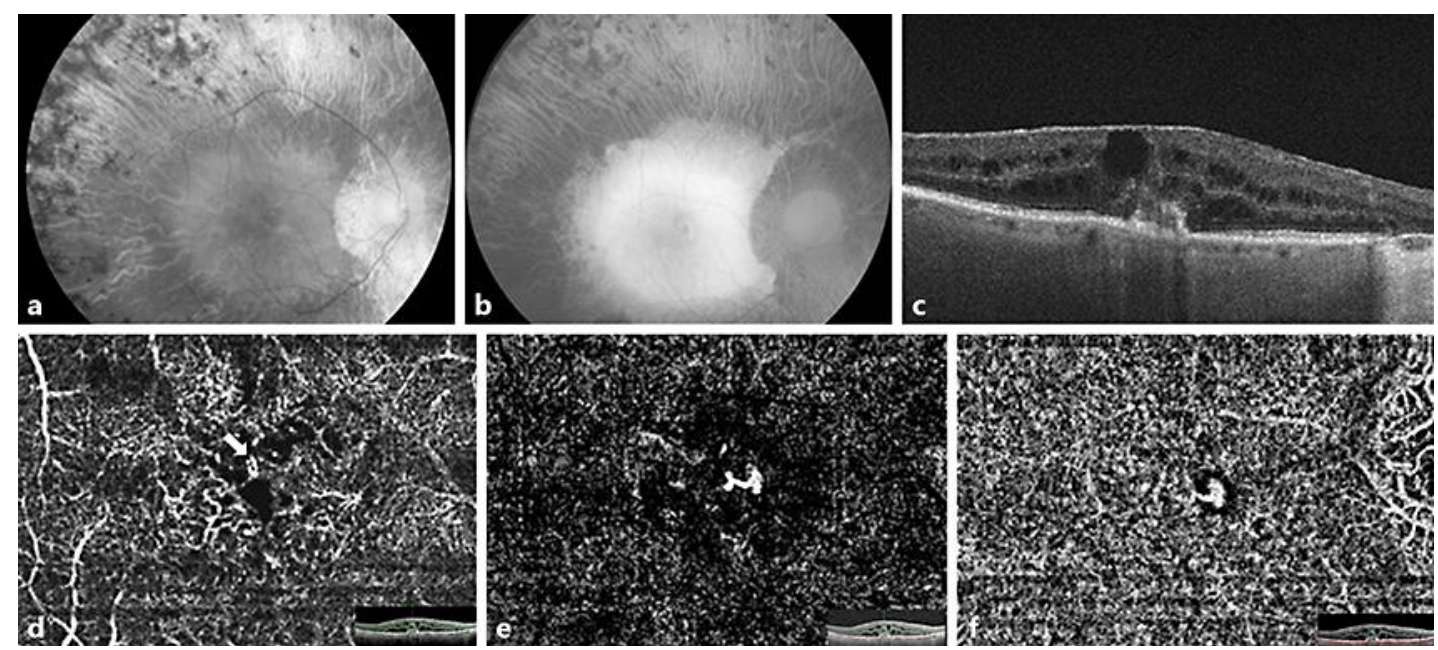

Fig. 1. a Monochromatic green fundus photograph of the right eye showing optic disk pallor, peripapillary atrophy, arteriolar narrowing, and typical bone spicules involving the mid-peripheral retina. b Fundus autofluorescence photograph of the right eye. Note the extent of the atrophy in the mid-periphery. c Spectral domain optical coherence tomography revealing a cystic macular edema associated with a disruption of the Bruch membrane/retinal pigment epithelium complex overlying a pigmentary epithelium detachment, with a vascular structure emanating from the deep capillary plexus, apparently connected with the subretinal pigment epithelium space. Also note the presence of an epiretinal membrane. $\mathbf{d}-\mathbf{f}$ Optical coherence tomography angiography images $(3 \times 3 \mathrm{~mm})$ and corresponding B-scans. d Optical coherence tomography angiography deep capillary plexus segmentation showing a high-flow vessel infiltrating the outer retinal layers (arrow). e Outer retinal layer segmentation showing a tuft-shaped high-flow lesion, characterized by a retinal-retinal anastomosis. $\mathbf{f}$ Choriocapillaris segmentation revealing the tuft-shaped lesion. 